\title{
Intelligent Space for Human Centered Robotics
}

\author{
Kazuyuki Morioka, Joo-Ho Lee and Hideki Hashimoto \\ Meiji University, Ritsumeikan University, University of Tokyo \\ Japan
}

\section{Introduction}

Robot systems for supporting human life are desired recently. For that purpose, the robots need to recognize a human living environment that changes dynamically. However, it is difficult to achieve it only with the sensors carried in a stand-alone robot. Then, the research field on the intelligent environments based on the sensor network has been expanding [Sato], [MacDorman]. The intelligent environments generally utilize many intelligent devices, such as computers and sensors in order to improve the performance of the robots. We proposed the intelligent space (iSpace) [Lee, (2002)] as the human-robot coexistent system. The Intelligent Space is designed to achieve a human-centred service by accelerating the physical and psychological interaction between human beings and robots. The iSpace exploits intelligent devices with processing and networking parts in addition to sensing images from colour CCD cameras. The iSpace with vision based intelligent devices achieves a contact-free wide-area location system without an additional burden to users. Vision based iSpace also has the possibility that the further information can be obtained depending

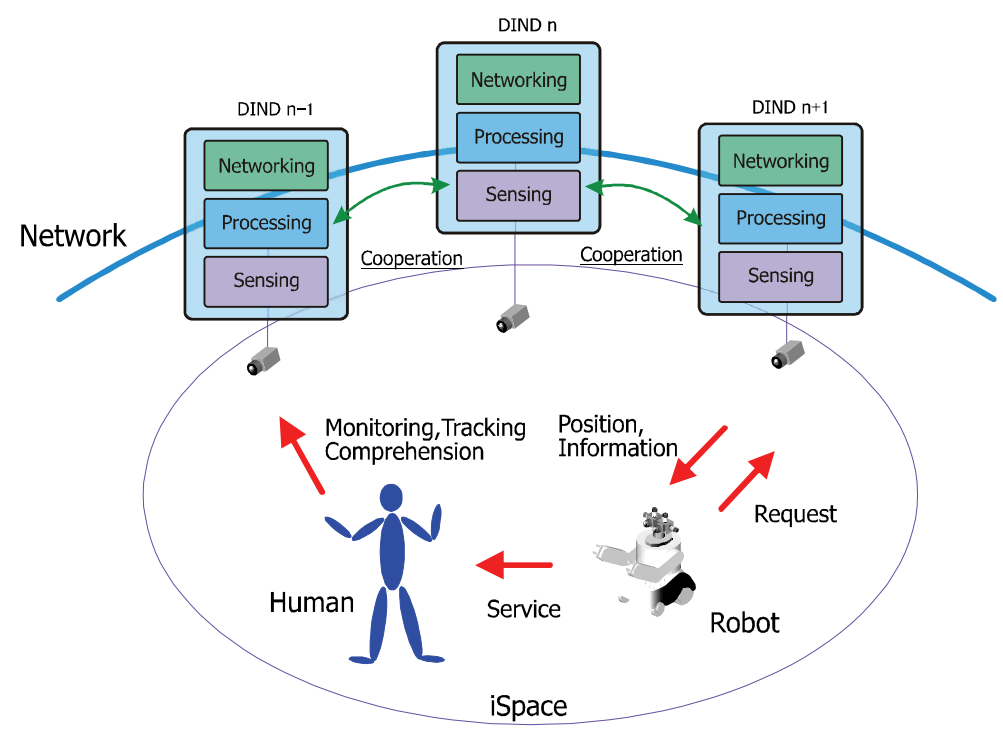

Fig. 1. Intelligent Space 
on image processing. Distributed vision devices offer promising prospects as the infrastructure in a human-centred robotic system for these reasons. The most important task of the sensing infrastructure in the iSpace is object tracking and localization of moving objects. Object tracking system in the iSpace is regarded as the multi-camera multi-object tracking system. There are two major problems in the multiple-camera multiple-object tracking system. The first problem is the traditional correspondence problem from frame to frame over time in the image sequence. The second is the object correspondence problem among different cameras in order to achieve seamless tracking. This paper introduces the vision based Intelligent Space and describes colour based target tracking with multiple vision sensors. The iSpace aim to achieve human-centred robotic systems with a tracking system based on vision sensors. To get close each other stably and physically is required for cooperative works between human beings and robots. One of solutions for achievement of such a human-centred robotic system is a human-following robot. A mobile robot for following human beings is also described as an application of tracking system in the Intelligent Space.

\section{Intelligent space}

\subsection{Concept}

The Intelligent Space is a space where many intelligent devices are distributed throughout the whole of the space, as shown in Fig. 1. These intelligent devices have sensing, processing and networking functions, and are named distributed intelligent networked devices (DINDs). One DIND consists of a CCD camera for acquiring space information, and a processing computer which has the functions of data processing and network interfacing. These devices observe the positions and behaviours of both human beings and robots coexisting in the iSpace. Based on the accumulated information, the environment as a system is able to understand the intention of human beings. For supporting humans, the environment/system utilizes machines including computers and robots.

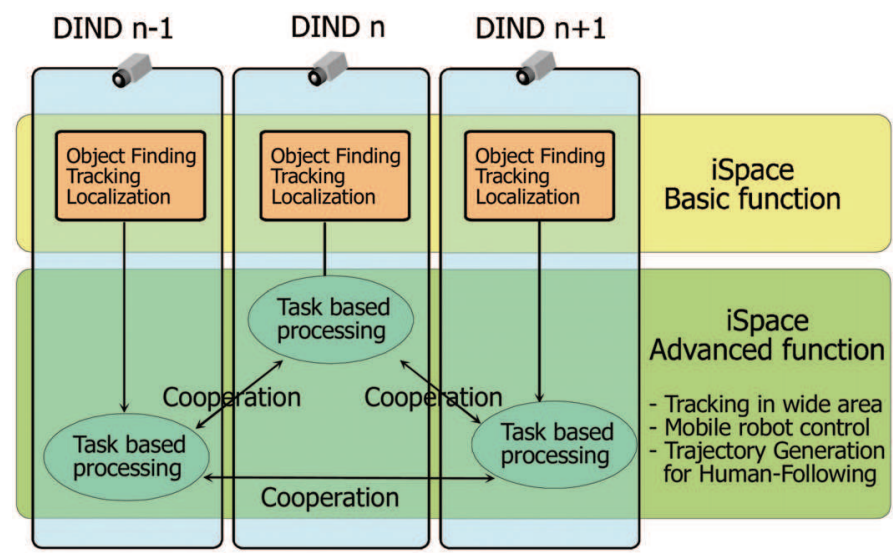

\section{Fig. 2. DIND Configuration}

The role of each DIND is separated into two parts as shown in Fig. 2. Each DIND should find human beings and robots as new target objects. Then, it tracks the target objects and gets location of them as basic functions independently of condition of other cameras, 
because of keeping the robustness and the flexibility of the system. On the other hand, the information acquired by each DIND is shared among the DINDs through the network communication system. At least, target tracking in wide area monitored by multiple DINDs must be achieved as the advanced function of DIND. The protocol and data structure between the DINDs should be decided for achievement of advanced functions. It also becomes easy to install new technology as new modules to the iSpace with the unified protocol and data structure between the DINDs and modules in advance. In the iSpace, distributed devices based system enables the achievement of a flexible system based on this feature. The human-following robot introduced in this chapter is a new module for the iSpace, as it is one of the applications located in a level higher than the module of target tracking and location system based on distributed devices.

\subsection{Intelligent space with vision based DIND}

There are two major problems in the multiple-camera multiple-object tracking system like the iSpace as described before. The first problem is the traditional correspondence problem from frame to frame over time in the image sequence. The second is the object correspondence problem among different cameras for seamless tracking. For solving the first problem, color model based object tracking algorithms, such as MeanShift algorithm, are effective with fast and robust object tracking. This paper also describes one of the tracking methods applied for the iSpace in the following section.

On the contrary, there are several approaches to solve the latter problem in the recent literatures. These approaches include feature matching, 3D information and etc. These are mainly problems of image processing. However, in order to solve multi-camera tracking problem, the other problem establishment as sensor system architecture is needed. Many DINDs are placed redundantly and randomly as a distributed vision sensor network in the iSpace. An appropriate camera for seamless tracking should be selected from many candidates according to a tracking capability of each camera. The following section introduces a handing over protocol for sharing the information among DINDs and tracking the targets in wide area.

Target position in the space is mainly communicated between the basic function and the advanced functions as shown in Fig.2. It is also possible to install new tracking algorithms in the part of basic functions by exploiting the target position as the lowest level interface between two functions.

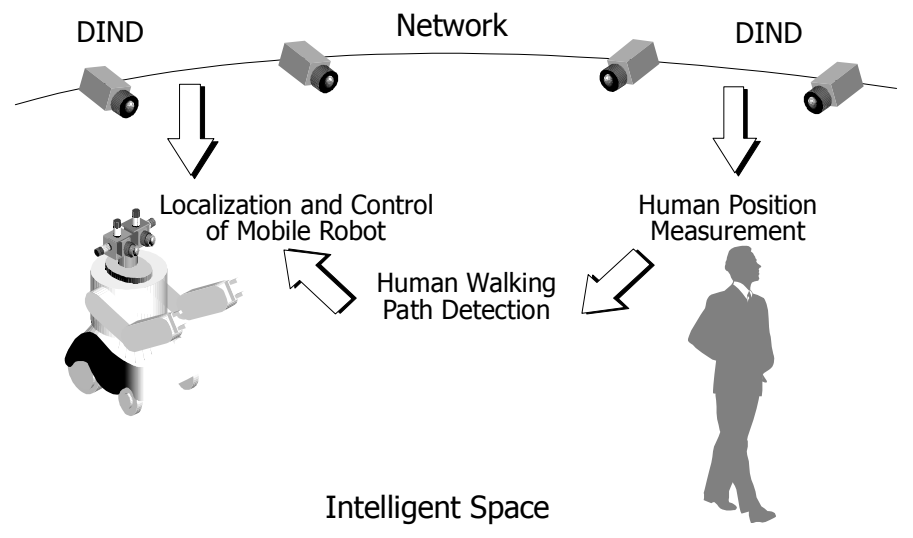

Fig. 3. Human-Following Robot in iSpace 


\subsection{Human centered robot in intelligent space}

A mobile robot is one of the physical agents for supporting human beings in the iSpace. In order for human beings and robots to coexist and to perform cooperative works, they have to interact closely. A solution for achievement of human-centred robotic system is a humanfollowing robot keeping a certain relative positional relationship between a human being and a robot. The human-following robot is able to provide human-centred services with following behaviour. In the case that a mobile robot accompanies a human being, the robot is able to easily acquire detailed information associated with a target person. It has been also reported that a close distance between a human being and a robot leads to mutual interactions [Sidenbladh]. Human-following robot needs several technologies, such as recognition of human beings, position estimation of human beings, and a control strategy for following human beings. In order to recognize human beings, most human-following robots developed before mounts many sensors, such as cameras, ultrasonic sensors and etc. These sensors detect the relative position from a mobile robot to a target human. The mobile robot in a literature [Sidenbladh] recognizes a human's skin colour using a camera, and traces a target human with pan-tilt control of a camera. Some of proposed human-following robots burden a target person with special equipments. It is not reasonable for a mobile robot to continue following a human being with avoiding other obstacles, without missing the target walking at a natural speed, since stand-alone robots have limitations in terms of recognition performance. Tracking system of the Intelligent Space is suitable as solutions of these problems. A mobile robot cooperates with multiple DINDs distributed in the environment. The DINDs recognize the target human and the mobile robot, and give control commands to the robot as shown in Fig.3. The mobile robot can receive necessary supports for human-following control from environmental sensors in iSpace.

\section{Tracking vision in intelligent space}

\subsection{Color based object tracking}

One of the object trackers in the DIND exploits colour histograms which represent the target feature. The object representation based on the colour histogram is relatively stable against deformation and occlusion. Recently, the tracking system based on mean shift algorithm is reported that it is suitable for the color region tracking [Comaniciu]. In this algorithm, weighted mean shift is used for multiple color region tracking. Weight is computed based on the color information of the object regions and background image as shown in Fig.4. In the research field of motion-based object tracking, one of the challenging tasks is how to deal well with the rapid movement of the object. An integrated method mean shift and Kalman filter has been proposed in the previous studies. It has proven to be efficient and relatively robust to the rapid movement of the object. In addition, this method has been compensated the weakness of mean shift tracker with Kalman filter. However, in case that the movement of a target is changed suddenly by collision with obstacles such a human, floor, and so on, the tracker loses the target object and there are few chances to recover. By adding changes to this algorithm, we were able to deal with the above problem. The dissimilarity of histograms between the model and the candidate region is expressed by a metric based on the Bhattacharyya distance. This measure has shown the benefit to achieve stable and efficient tracking in our research. In order to localize the target object, the mean shift procedure and the Kalman filter is used. In this research, we added feedback loop after 
the mean shift procedure. Tracking experiments in which our method coped with the sudden change of the object movement are presented.

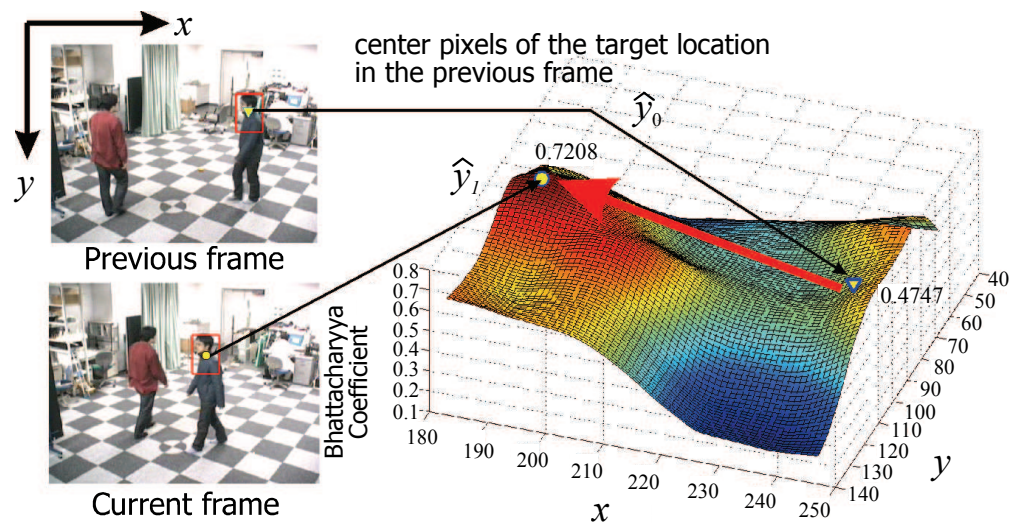

Fig. 4. MeanShift Tracker

A new approach toward target tracking [Morioka, (2007)] is shown in Fig.5. In conventional algorithm using mean shift and Kalman filter, the target location is decided through the "path1" on the Fig.5. To handle the sudden change of the movement, we added feedback loop "path2" after the mean shift process. In this algorithm, regular target localization process goes through "path1". In case that unexpected change of the movement happened and the tracker lost the object, this estimation depends on the Bhattacharyya distance, the second localization procedure starts from the position of the target in the previous frame and with the size of the region is temporarily expanded through "path2". The magnification

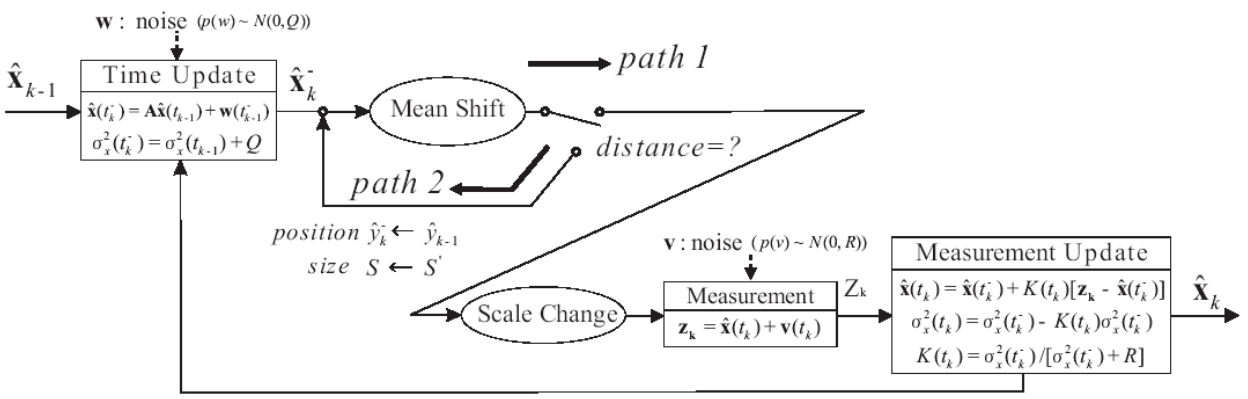

Fig. 5. Hybrid Tracking Algorithm

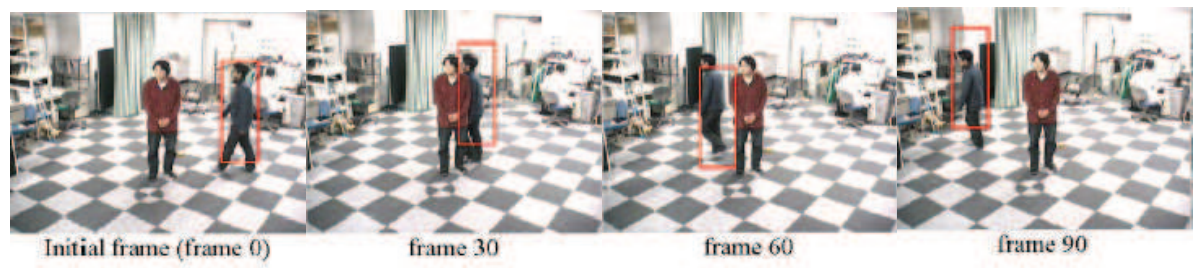

Fig. 6. Tracking Results 
ratio is application dependent. We use an area less than twice the target area and adopt scalar value of the velocity vector of the target object. The means shift tracker has the weakness. That is unable to cope with rapid movement of object because the object is not included in the predicted area with Kalman filter. On the other hand, the mean shift tracker shows stability and efficiency in case that the target region overlaps with the target object. The function of "path2" is to operate the mean shift. The greatest advantage of our hybrid algorithm is that continuation of the object tracking is possible efficiently, without applying load to a system. Fig.6 shows the experimental result of human tracking with the proposed tracker. In this experiment, HSV colour space is used for configuration of colour histogram. Each axis of the HSV colour space is divided to 32 bins. Image coordinates of tracked objects are converted to world coordination based on camera parameters by using a set of two DINDs. World coordinates can be obtained from one DIND in the case that the height of target object is known.

\subsection{Handing over for seamless tracking of mobile robot}

A DIND is an independent device, and its functions, including tracking and localization of target objects, are performed completely within it. Especially, tracking and localization of a mobile robot is the most important task for achievement of human-centred robotic system. In addition to localization, a DIND sends control signals based on localization results to a target mobile robot. Thus, if a mobile robot is moving in the area a DND is monitoring, the robot is guided without any difficulty. However, to guide robots in a wider area, which a single DIND cannot cover, we define the DIND that has the control authority of a robot as a dominant DIND for the robot. When a robot moves from an area to a different area, the robot's dominant DIND should be automatically changed. This is called "handing over of control authority" [Lee, (2004)]. A dominant DIND has the control authority of the robots and at one time only a single dominant DIND exists for each robot. Therefore, the control authority should be smoothly handed over to the next DIND at the proper time and location. To solve this problem, a reliability rank is devised. High reliability stands for that a DIND can guide a robot robustly and precisely. Generally, the area near a DIND and the centre area of an image captured by a DIND, have the highest reliability rank; while the boundary of the image and the area far from the DIND have the lowest reliability since a camera is adopted as a sensor for a DIND. Fig.7 shows that the monitored area is divided into several areas and the actual reliability ranks are determined.

Fig. 8 shows a process for handing over of the control authority of a target robot. Circles indicate the monitoring areas of cameras. The monitoring areas of dominant DINDs are coloured. First, the dominant DIND requests information from the other DINDs about the reliability rank of the target robot. The other DINDs reply with their reliability rank concerning the robot and the current dominant DIND. The dominant DIND compares these values with its own rank. If the other DIND has a higher rank than its own rank, then the current dominant DIND transfers the authority of control to the other DIND, which has the higher rank. Then the new dominant DIND tracks and controls the robot.

Fig.9 shows the results of the handing over among three DINDs. $(x, y)$ coordinates of a tracked mobile robot in world coordinate system are plotted in this figure. In this experiment, a simple colour tracking is exploited for tracking in a single DIND [Appenzeller]. At the beginning, the mobile robot was located in the monitoring area of 
DIND 3. Three kinds of signs of Fig. 9 show the ID of DIND which the mobile robot was tracked by. According to the position of the robot, the dominant DIND changed in the order of DIND 3, 1, 2. It turned out that the mobile robot continued to be tracked, even when it's dominant DIND changed. The estimated positions of the mobile robot were discontinuous at the point where the dominant DIND changed. This was caused by position measurement error resulting from camera calibration and image processing. However, the error hardly influenced the control of the mobile robot because of the proposed control method as follows.

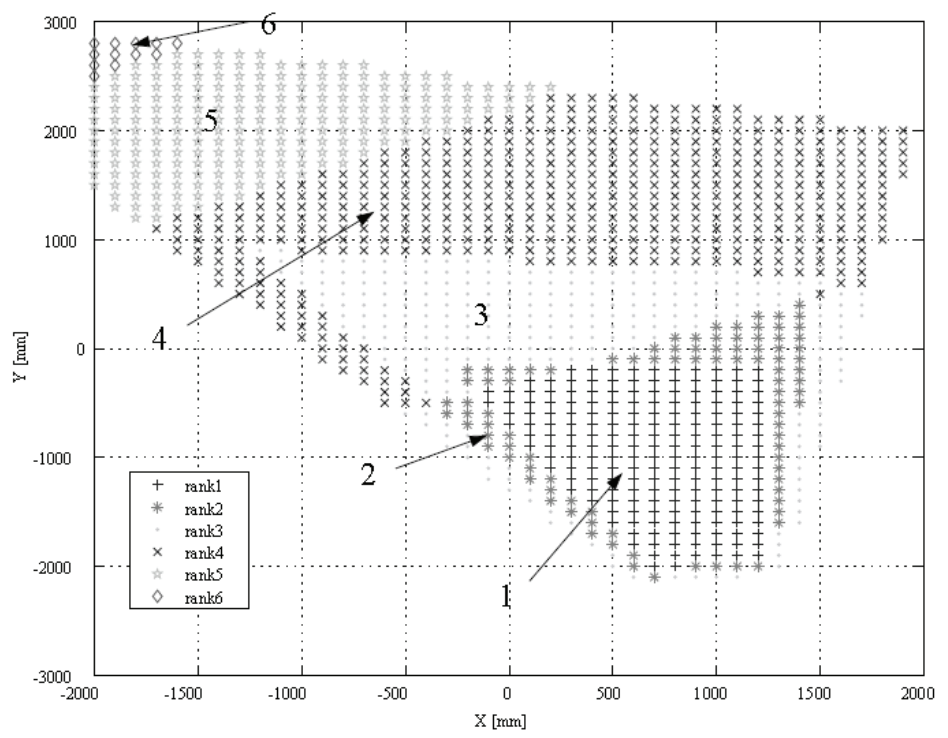

Fig. 7. Reliability Map of Monitoring Area
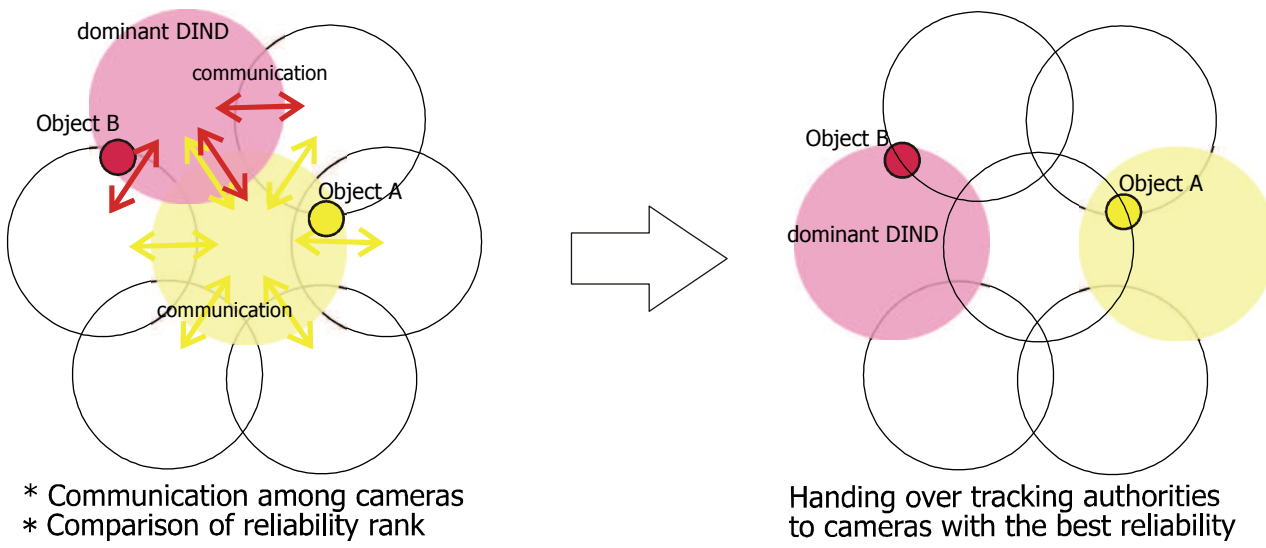

Handing over tracking authorities to cameras with the best reliability

Fig. 8. Handing Over 


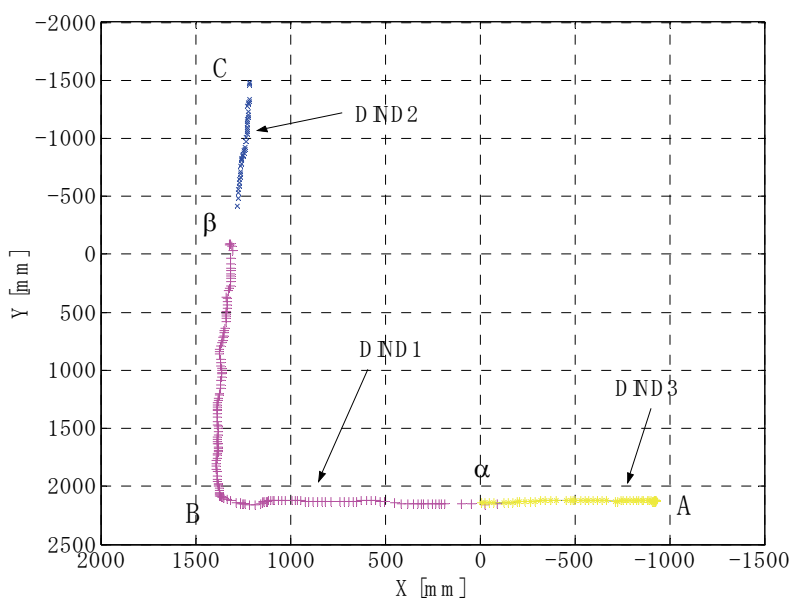

Fig. 9. Tracking Result with Handing Over Protocol

\section{Application - human-following mobile robot}

\subsection{Human-following mobile robot in iSpace}

In this section, a human-following mobile robot is introduced as an application of vision based iSpace. Several effective features are expected from the human-following robot as a physical agent in iSpace. The first feature is that human following can be realized without depending on performances of mobile robots. Intelligence of not a robot itself but the iSpace is mainly used for navigation of a mobile robot in iSpace [Lee, (2002)]. For this reason, it is possible to perform human-following operations in iSpace with any type of mobile robot, even though robots lack sensors and intelligence. The second feature is that human following is possible even in a complicated environment. The iSpace usually acquires information about target objects such as human beings, robots and obstacles with cooperation of multiple DINDs. Since mobile robots are managed by DINDs from the environment side, the iSpace is able to generate each robot's path such that they do not collide with each other. Therefore, the robots are able to move smoothly, even if they are in a complicated environment. The third feature is that the iSpace can include various functions; behaviour-based control [Szemes] and spatial memories [Niitsuma] and etc. When these functions and the human-following robot system are combined, more meaningful actions of mobile robots will be achieved. When a robot with such advanced functions is in an ordinary space, the robot is able to afford similar functions as the robots in the iSpace. However, its serviceable area is limited to a range near the robot. It is difficult to provide suitable services for people in far from the robot. In such a case, a human being is required to approach the mobile robot in order to inform his/her requirements and to receive services from the robot. A human-following mobile robot in the iSpace is one of the best solutions as a human-centred robotic system for these reasons.

\subsection{Control and system configuration}

A differential wheel velocity-type mobile robot is used for the human-following robot because this type of robot has a simple and compact structure. It is suitable for a physical 
agent that has to interact with human beings in complicated environments. Our mobile robot is based on the Pioneer2-DX. This mobile robot is connected to the DIND network via wireless LAN. Since the height of the mobile robot is already known, the position of a mobile robot is reconstructed from one camera image.. Positions and headings of a mobile robot are measured with tracking of three colour markers mounted on the top of a robot.

A tracking control is performed to a mobile robot in order to follow a human being. Many studies have been performed in the field of the tracking control. In the control of a nonholonomic mobile robot, Brockett's theorem proved that a smooth state feedback law for an asymptotically stable to one point of the state space does not exist [Nakamura]. In recent years, various closed-loop control systems which can overcome the feedback stabilization impossibility of Brockett's theorem have been proposed. In the iSpace, since a human walking trajectory is newly generated in every step, it can be considered as a function of time. Therefore, the application of tracking control is effective. However, the target trajectory for control of a mobile robot is limited to continuous and smooth ones in most cases. A human-following robot should be able to track actual human walking trajectories, including abrupt changes in velocity and direction. A human-following robot with a conventional tracking control may lose stable tracking of a target person. A tracking control method in the iSpace for following human beings should be newly proposed.

The estimated human position data is not proper control input required for a mobile robot to follow a human being since estimated position data contains errors in the form of calibration error and image processing error. When the heads of humans are measured by vision sensors in a short sampling period, their velocity and direction also change drastically. In addition to unstableness of position data measured by the iSpace, the direction and velocity of human walking sometimes changes suddenly and unpredictably. In this case, the mobile robot may fail to follow the target human and lose stable movement by a sudden change in a velocity input. It is considered that a mobile robot cannot follow the target human with conventional control law derived only from the distance between the target human and the mobile robot. There are fundamental differences between a mobile robot and a human being in the level of motion. A human is able to move freely by foot. However, a differential wheel velocity type mobile robot with nonholonomic constraints cannot move as freely as does a human. To trace a human who walks freely, a control strategy that overcomes the limitations of nonholonomic constraints is needed. This chapter introduces a control law with a virtual spring model for absorbing the kinematic difference between the human and the mobile robot [Morioka, (2004)]. The proposed control law is derived from an assumption that a human being and a mobile robot are connected by a virtual spring as shown in Fig.10. An input velocity to a mobile robot is generated on the basis of an elastic force of a virtual spring in this model. In the proposed control system, the virtual spring works as a low pass filter and absorbs adverse fluctuations. The proposed virtual spring model is able to absorb the gap between a motion of human being and that of the mobile robot.

DIND software is actually implemented with configuration as shown in Fig. 11. A program for DIND basic functions is always running in each DIND. This basic program includes image capture, image processing for target tracking, and reconstruction of 3D coordinate. In the case of 3D reconstruction of a target with unknown height, two cameras are combined as a sensor part of DIND. A DIND basically makes connections to other DINDs with the client/server method of UDP protocol. A program for DIND basic functions in each DIND is always running for obtaining target measurements. Server programs for advanced 
functions are also running in order to receive requests for passing the control authority from other DINDs or to provide measurement positions of target human beings. Client programs are executed according to the situation needed for advanced functions. For example, client for advanced task \#0 will start in the case that DIND requests handing over to other DINDs. The client for advanced function \#1 for making a connection to the mobile robot become active if DIND has a control authority for a human-following robot. It provides the velocity inputs for a human-following mobile robot. The client for advanced function \#2 is also executed for obtaining target human positions in human-following task.

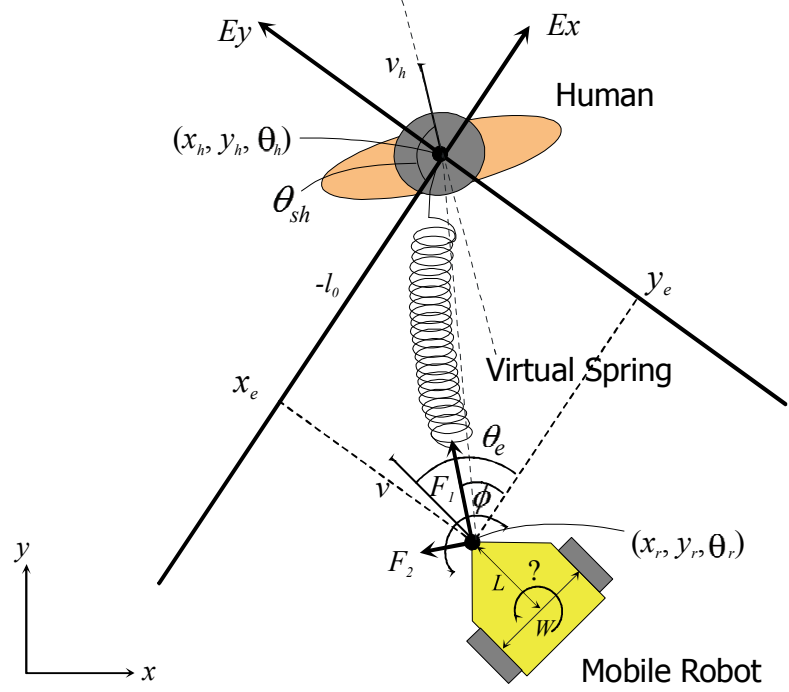

Fig. 10. Virtual Spring Model

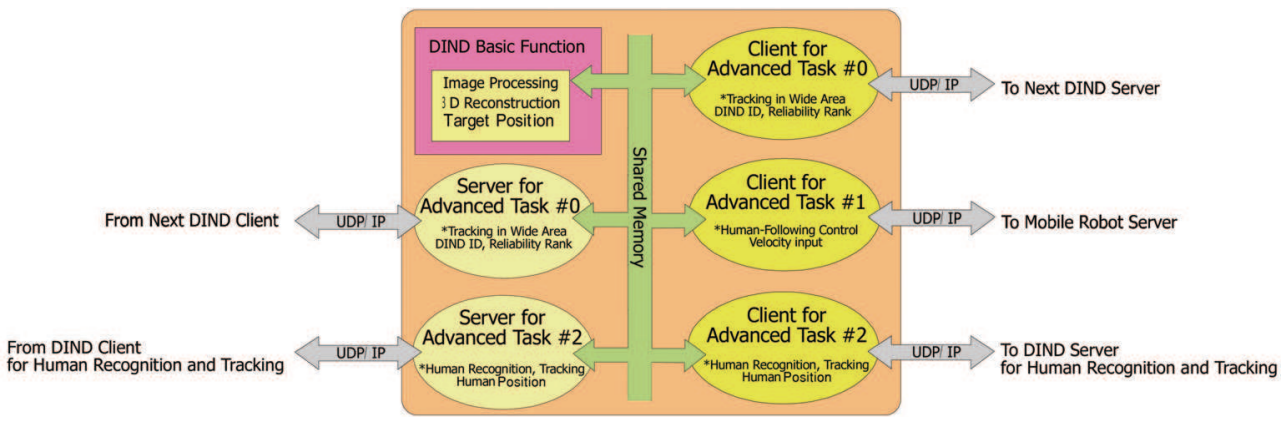

Fig. 11. Hybrid Tracking Algorithm

\subsection{Experiment}

The results of the experiment are shown in Fig.12. In this experiment, four DINDs for robot control and one DIND with two cameras for human tracking are placed in the iSpace. A server program for human recognition and tracking are active in the DIND for human tracking. The programs for advanced tasks except to a server for human recognition and 
tracking become active in the DINDs for robot control according to the situations. In this experiment, a simple colour tracking [Appenzeller] is applied for basic functions. DINDs for robot control have a colour model of a mobile robot, and a DIND for human tracking just has a skin colour model of human beings in advance. A human walked along a hexagonal path. The mobile robot followed him along the course plotted by four kinds of signs in Fig.13. Four signs in Fig.13 show that the dominant DIND for the mobile robot changed according to the handing over protocol. In spite of the unstable human position estimation, the mobile robot was controlled smoothly. The low-frequency fluctuations in the human trajectory were cut off by the virtual spring and the robot was controlled smoothly. It is found from the results that the proposed algorithm allows the robot to follow the target without excessive motions. In Fig.12, the dashed line represents the position measured by the encoders in the robot. The dashed line deviates significantly from the trajectory of the robot measured by the DINDs. This is mainly caused by slips of the wheels and the wrong internal parameters of the robot. It is also an advantage for the human-following robot realized in the iSpace to be robust against various errors that disturb the robot's navigation. Although this human path is simple from a global point of view, complex motions, such as variations in the velocity and direction of humans are included in the human's actual movement. The validity of the human-centred robotic system based on the iSpace was verified from this experimental result.

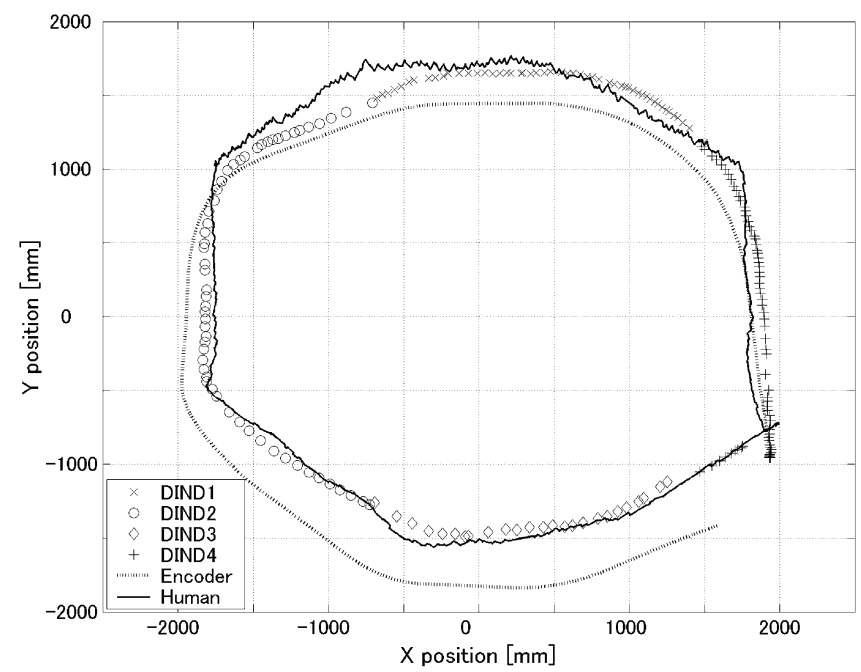

Fig. 12. Experimental Results of Human-Following

\section{Conclusion}

In this book chapter, the Intelligent Space for achievement of human-centred robotic system was presented. The positions of target objects in the iSpace should be measured with multiple DINDs installed in a wide area. This chapter introduced a hybrid tracking algorithm including MeanShift and Kalman filter for object tracking in one DIND. To track target objects in a wide area and control mobile robots based on environmental measurement, cooperation of the DINDs, effective communication and role assignment are 
required. The handing-over protocol for a mobile robot control was explained. Finally, a human-following mobile robot was introduced as an application of the iSpace for a humancentred robotic system. A human-following robot is based on the measurement infrastructure of the iSpace with multiple DINDs. A proposed control with a virtual spring model was implemented as a new module in each DIND. The result shows that humanfollowing is easily achieved in the iSpace. Future studies will involve applying this system to complex environments where many people, mobile robots and obstacles coexist. It is necessary to investigate the influence of a human-mobile robot which maintains a fixed distance between a robot and a target human with introducing knowledge of cognitive science and social science.

\section{References}

Appenzeller, G.; Lee, J.-H.; \& Hashimoto, H. (1997). Building Topological Maps by Looking at People: An Example of Cooperation between Intelligent Space and Robots, Proc. of IEEE/RSJ International Conference on Intelligent Robots and Systems, Vol.3, pp.13261333, ISBN 0-7803-4119-8, Grenoble, France

Comaniciu, D.; Ramesh V. \& Meer, P. (2003). Kernel-Based Object Tracking, IEEE Trans. on Pattern Analysis and Machine Intelligence, Vol.25, No.5, pp.564-577, ISSN 0162-8828

Lee, J.-H \& Hashimoto, H. (2002). Intelligent Space - concept and contents. Advanced Robotics, Vol.16, No.3, pp.265-280, ISSN 0169-1864

Lee, J.-H.; Morioka K.; Ando N. \& Hashimoto H. (2004). Cooperation of Distributed Intelligent Sensors in Intelligent Environment. IEEE/ASME Trans. on Mechatronics, Vol.9, No.3, 2004, pp.535-543, ISSN 1083-4435

MacDorman, K. F.; Nobuta, H.; Ikeda, T.; Koizumi, S. \& Ishiguro, H. (2004). A memorybased distributed vision system that employs a form of attention to recognize group activity at a subway station, Proc. of IEEE/RSJ International Conference on Intelligent Robots and Systems, pp.571-576, ISBN 0-7803-8464-4, Sendai, Japan

Morioka, K.; Lee J.-H. \& Hashimoto H. (2002). Human- Following Mobile Robot in a Distributed Intelligent Sensor Network. IEEE Trans. on Industrial Electronics, Vol.51, No.1, 2004, pp.229-237, ISSN 0278-0046

Morioka K.; Lee, J.-H.; Kuroda, Y. \& Hashimoto, H. (2007). Hybrid Tracking Based on Color Histogram for Intelligent Space. Journal of Artificial Life and Robotics, Vol.11, No.2, pp.204-210, ISSN 1433-5298

Nakamura, Y. (1993). Nonholonomic robot system, Part 2: Motion planning under kinematic nonholonomic constraints. Journal of Robotics Society of Japan, vol. 11, no. 5, pp. 655662, ISSN 0289-1824

Niitsuma. M.; Hashimoto, H. \& Hashimoto, H. (2007). Spatial Memory as an Aid System for Human Activity in Intelligent Space. IEEE Trans. on Industrial Electronics, Vol.54, No.2, pp.1122-1131, ISSN 0278-0046

Sato, T.; Harada, T. \& Mori, T. (2004). Environment-Type Robot System "Robotic Room" Featured by Behavior Media, Behaviour Contents, and Behavior Adaptation. IEEE/ASME Trans. on Mechatronics, Vol.9, No.3, pp.529-534, ISSN 1083-4435

Sidenbladh, H. \& Kragic, D. \& Christensen H. I. (1999). A person following behavior for a mobile robot, Proc. 1999 IEEE Int. Conf. Robotics and Automation, Vol.1, pp. 670-675, ISBN 0-7803-5180-0, Detroit, USA

Szemes, P.; Hashimoto, H. \& Korondi, P. (2005). Pedestrian-behavior-based mobile agent control in intelligent space. IEEE Trans. on Instrumentation and Measurement, Vol.54, No.6, pp.2250-2257, ISSN 0018-9456 


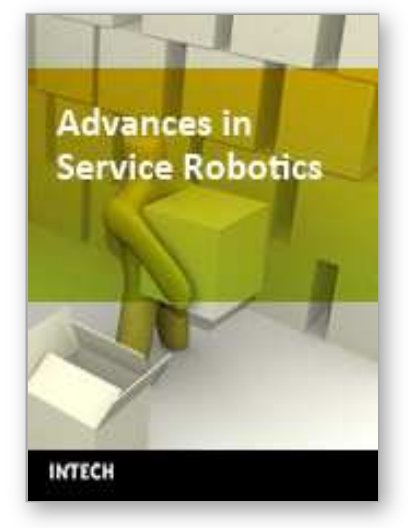

\author{
Advances in Service Robotics \\ Edited by Ho Seok Ahn
}

ISBN 978-953-7619-02-2

Hard cover, 342 pages

Publisher InTech

Published online 01, July, 2008

Published in print edition July, 2008

This book consists of 18 chapters about current research results of service robots. Topics covered include various kinds of service robots, development environments, architectures of service robots, Human-Robot Interaction, networks of service robots and basic researches such as SLAM, sensor network, etc. This book has some examples of the research activities on Service Robotics going on around the globe, but many chapters in this book concern advanced research on this area and cover interesting topics. Therefore I hope that all who read this book will find lots of helpful information and be interested in Service Robotics. I am really appreciative of all authors who have invested a great deal of time to write such interesting and high quality chapters.

\title{
How to reference
}

In order to correctly reference this scholarly work, feel free to copy and paste the following:

Kazuyuki Morioka, Joo-Ho Lee and Hideki Hashimoto (2008). Intelligent Space for Human Centered Robotics, Advances in Service Robotics, Ho Seok Ahn (Ed.), ISBN: 978-953-7619-02-2, InTech, Available from: http://www.intechopen.com/books/advances_in_service_robotics/intelligent_space_for_human_centered_robot ics

\section{INTECH}

open science | open minds

\section{InTech Europe}

University Campus STeP Ri

Slavka Krautzeka 83/A

51000 Rijeka, Croatia

Phone: +385 (51) 770447

Fax: +385 (51) 686166

www.intechopen.com

\section{InTech China}

Unit 405, Office Block, Hotel Equatorial Shanghai

No.65, Yan An Road (West), Shanghai, 200040, China

中国上海市延安西路65号上海国际贵都大饭店办公楼405单元

Phone: +86-21-62489820

Fax: $+86-21-62489821$ 
(C) 2008 The Author(s). Licensee IntechOpen. This chapter is distributed under the terms of the Creative Commons Attribution-NonCommercialShareAlike-3.0 License, which permits use, distribution and reproduction for non-commercial purposes, provided the original is properly cited and derivative works building on this content are distributed under the same license. 